\title{
Influencias psicosocioculturales sobre los sintomas psicopatológicos en comunidad abierta: desigualdades ecosistémicas
}

\author{
Ana Olivia Ruiz-Martínez; Norma Ivonne González-Arratia López Fuentes; Sergio González-Escobar; \\ Yessica Paola Aguilar-Montes de Oca; Martha Adelina Torres-Muñoz
}

How to cite this article: Ruiz-Martínez, A.O., González-Arratia López Fuentes, N.I., González-Escobar, S., Aguilar-Montes de Oca, Y.P. \& Torres-Muñoz, M.A. (2020). Psycho-sociocultural influences on psychopathological symptoms in the open community: ecosystemic inequalities Acta Colombiana de Psicología, 23(1), 169-180. doi: http://www.doi.org/10.14718/ ACP.2020.23.1.9

Recibido, mayo 8/2018; Concepto de evaluación, septiembre 24/2018; Aceptado, abril 4/2019

\author{
Ana Olivia Ruiz-Martínez \\ Universidad Autónoma del Estado de México, Zumpango, México \\ ORCID: https://orcid.org/0000-0002-9286-5946 \\ Norma Ivonne González-Arratia López Fuentes \\ Universidad Autónoma del Estado de México, Toluca, México \\ ORCID: https://orcid.org/0000-0003-0497-119X \\ Sergio González-Escobar \\ Universidad Autónoma del Estado de México, Atlacomulco, México \\ ORCID: https://orcid.org/0000-0002-9283-6985 \\ Yessica Paola Aguilar-Montes de Oca \\ Universidad Autónoma del Estado de México, Toluca, México \\ ORCID: https://orcid.org/0000-0002-4427-4973 \\ Martha Adelina Torres-Muñoz \\ Universidad Autónoma del Estado de México, Toluca, México \\ ORCID: https://orcid.org/0000-0002-8481-0048
}

\begin{abstract}
Resumen
Los problemas de salud mental en la población general tienden a estar relacionados con determinantes sociales que también influyen en la inequidad sanitaria. Sin embargo, estos determinantes suelen tenerse en cuenta solo en la población clínica y pasan inadvertidos en el ámbito comunitario. Teniendo esto en cuenta, el propósito del presente estudio fue identificar los factores psicosocioculturales que influyen en la presencia de los síntomas psicopatológicos en población abierta. Para esto, participaron 229 mujeres y hombres, adultos, mexicanos, a quienes se les aplicaron dos instrumentos de autorreporte para evaluar sus síntomas psicopatológicos, el SCL 90-R y el Cuestionario de Factores Asociados a la Salud. Los resultados muestran diferencias entre hombres y mujeres tanto en los síntomas como en los factores evaluados, en donde las mujeres resultaron ser las más desfavorecidas. Los modelos predictivos señalan que en las mujeres los factores que predicen la sintomatología son los pensamientos conflictivos, la violencia intrafamiliar, la falta de confianza en sus capacidades y la discriminación de género; mientras que en los hombres fueron la violencia intrafamiliar, la pobreza y un menor bienestar. Se concluye que los modelos psicosociales y ecosistémicos permiten comprender que los factores psicosocioculturales influyen sobre los síntomas incipientes de población comunitaria, con vías diferentes tanto para las mujeres como para los hombres. Con esto se demuestra que el contexto imprime un impacto diferencial en la salud mental.

Palabras clave: salud mental, determinantes sociales, género, violencia intrafamiliar.
\end{abstract}

\footnotetext{
Camino viejo a Jilotzingo s/n, Valle Hermoso, Zumpango, Estado de México. C. P.. 55600. Tel.: 015919172702. ana_olivi@yaho.com.mx
} 


\title{
Psycho-sociocultural influences on psychopathological symptoms in the open community: ecosystemic inequalities
}

\begin{abstract}
Mental health problems in the general population tend to be related to social determinants which also influence health inequity. However, these determinants are usually taken into account only for clinical populations and often go unnoticed at the community level. The purpose of the present study was to identify the psycho-socio-cultural factors that influence the presence of psychopathological symptoms in the open population. In this study, 229 women and men, Mexican adults, participated. Two self-report instruments were used: the SCL 90-R to evaluate psychopathological symptoms, and the Questionnaire of Factors Associated with Health. The results show differences between men and women, both in the symptoms and in the factors evaluated, where women are more disadvantaged. Predictive models indicate that in women the factors predicting the symptomatology are: conflicting thoughts, domestic violence, lack of confidence in their abilities and gender discrimination; while in men they were: family violence, poverty and lower well-being. It is concluded that psychosocial and ecosystemic models enable to understand that psycho-socio-cultural factors influence the presence of incipient symptoms in the community population, following different paths for women and men, showing that the context has a differential impact on mental health.

Key words: mental health, social determinants, gender, intra-family violence.
\end{abstract}

\section{Introducción}

Actualmente, en el ámbito de la salud se reconoce que el proceso de salud-enfermedad es dinámico e integral, por lo que no basta con atender la salud física, sino que también debe incluir la salud mental, entendida como el estado de bienestar y de adaptación que le permite a la persona desarrollarse, resolver sus problemáticas y contribuir a la sociedad (Rodríguez, 2009). Esta concepción de salud mental se relaciona con el bienestar psicológico, que hace referencia a la percepción de satisfacción, felicidad y calidad de vida (Alvarado et al., 2013), y está en contraposición con el malestar psicológico, que tiende a asociarse a inquietudes, problemas, desasosiego e insatisfacciones que suelen manifestarse en síntomas psicopatológicos y que pueden llegar a acrecentarse hasta alcanzar los criterios clínicos de los trastornos mentales.

Dada la naturaleza multifactorial del proceso de saludenfermedad, es necesario reconocer que la presencia de la psicopatología no ocurre de manera individual ni asilada de su entorno, sino que en ella influyen elementos individuales, familiares y determinantes socioculturales del ecosistema que favorecen uno u otro extremo de este continuo. Respecto a esto, la Organización Mundial de la Salud (OMS, 2009) señaló que la distribución desigual del poder, de los ingresos, de los bienes y de los servicios influye en gran medida sobre las condiciones de vida, sobre todo en aspectos como la atención sanitaria, la educación, la vivienda, el trabajo y el medio ambiente. Teniendo esto en cuenta, los determinantes de la salud cobran una gran importancia, ya que se reconoce que la morbilidad y la mortalidad están íntimamente ligadas con la estructura social en la que vive la persona, donde se incluyen influencias tanto económicas como políticas y sociales, principalmente cuando imprimen una marcada inequidad sanitaria.

De esta manera, se puede decir que la salud mental está influenciada por una compleja interrelación de condiciones socioculturales —en donde destacan la pobreza, la urbanización desordenada, la carencia de vivienda y la falta de educación - que dependen de las economías y políticas gubernamentales nacionales e internacionales, de modo que estas instituciones serían responsables, en gran medida, de la inequidad y de la injusticia de la distribución de los recursos; factores que favorecen la presencia de los trastornos mentales en la población general (OMS, 2003, 2013; Organización Panamericana de la Salud [OPS], 2018a).

Según la OPS (2018b), en el ámbito internacional se reconoce que los trastornos mentales representan el $12 \%$ de la carga global de enfermedades; que en América Latina y el Caribe representan el $19 \%$ de los años de vida ajustados en función de la discapacidad (AVAD); y que los principales trastornos que se presentan en la población general son la depresión (3.4\%), la ansiedad (2.1\%) y los trastornos por dolor (2.0\%), los cuales, a su vez, son los que más años perdidos por discapacidad generan (APD). Asimismo, de acuerdo con la OPS (2017), la prevalencia de los trastornos mentales en adultos —en los últimos 12 meses - oscila entre el $18.7 \%$ y el $24.2 \%$, principalmente con trastornos de ansiedad (9.3\% al 16.1\%), afectivos (7.0\% al 8.7\%) y por abuso de sustancias (3.6\% y $5.3 \%$ ). Cabe destacar que en estas cifras de prevalencia se encuentran variaciones por género, ya que las mujeres presentan mayor depresión $\mathrm{y}$ ansiedad, mientras que los hombres presentan mayor consumo de alcohol (Kohn et al., 2005); y, en el caso de 
la depresión mayor, su prevalencia en mujeres oscila entre el $4.0 \%$ y el $10.3 \%$, mientras que en los hombres va del $2.8 \%$ al $6.9 \%$, con mayor medida en la vejez (Luppa et al., 2012).

Específicamente, en la región de las Américas —caracterizada por la inequidad entre sus habitantes - México ocupa un lugar intermedio en cuanto a la carga de los trastornos mentales, ya que estos presentan un $16 \%$ del AVAD y el $33 \%$ de AVP (OPS, 2018b). Acá, un referente obligado es la Encuesta Nacional de Epidemiología Psiquiátrica (ENEP) de 2003, en la cual se reporta la presencia de trastornos mentales en población abierta con $28.6 \%$ a lo largo de la vida, $13.9 \%$ en el último año y $5.8 \%$ en el mes reciente, donde, de igual forma, destacan los trastornos ansiosos y depresivos en las mujeres, y los de consumo de sustancias en los hombres (Medina-Mora et al., 2003).

Ahora bien, al considerar los síntomas psicopatológicos en población abierta se ha encontrado una prevalencia en adultos que oscila entre el $14.1 \%$ y el $16.3 \%$ en mujeres y de $6.7 \%$ a $14.4 \%$ en hombres (Ruiz, González, González, Aguilar \& Torres, 2018). Y, en particular, con respecto a las variaciones según el género, en algunos estudios se ha señalado que las mujeres obtienen mayores puntuaciones en todas las dimensiones del SCL-90-R, excepto en psicoticismo (Alvarado, Sandín, Valdez-Medina, González-Arratia \& Rivera, 2012; Ruiz et al., 2018), mientras en otros las mujeres solo obtienen mayores puntuaciones en somatización, depresión y sensibilidad interpersonal (Cruz, López, Blas, González \& Chávez, 2005). Esta inconsistencia también se ha encontrado en poblaciones más jóvenes, ya que en algunos estudios se reportan diferencias solo en somatización, depresión, trastorno obsesivo-compulsivo, sintomatología total y síntomas positivos (Rivera, Caballero, Pérez \& Montero, 2013), mientras que en otros no se encuentran diferencias por género en ninguno de los síntomas (SCL90-R) con muestras de jóvenes de 16 a 19 años (Kröninger \& Grevenstein, 2013).

Cabe señalar que los datos de prevalencia en población abierta suelen ser inferiores a los presentados en poblaciones que acuden a los servicios de salud por atención médica o psiquiátrica, y que, además, es importante considerar el instrumento utilizado, ya que, por ejemplo, Espinosa, Orozco e Ybarra (2015), utilizando la escala de Goldberg en hombres que solicitaron atención médica, encontraron la presencia de síntomas ansiosos en el $57 \%$, y de síntomas depresivos en el $51 \%$.

Como se puede observar, los factores que influyen en la presencia de la psicopatología van desde aspectos demográficos - como el género- hasta aspectos macrosociales - como las políticas y la economía- y, en este sentido, la literatura internacional señala que dichos problemas se acentúan en población femenina, con bajos ingresos y en personas pertenecientes a pueblos originarios (Kisely et al., 2017; OPS, 2018a). Teniendo esto en cuenta, la población mexicana tendría un grado de vulnerabilidad importante, ya que en ella destacan aspectos como los sucesos estresantes, la violencia de género (Medina-Mora et al., 2005a), la edad de inicio de la psicopatología, los bajos ingresos, un gran número de mujeres separadas, viudas y divorciadas (Medina-Mora et al., 2005b), la migración (Breslau et al., 2007), la educación inconclusa (Lee et al., 2009), y altos niveles de abuso y disfunción familiar (Benjet, Borges \& Medina-Mora, 2010).

Por otra parte, con respecto a los aspectos económicos, se ha encontrado que la carencia se asocia con mayor severidad en los trastornos mentales (Medina-Mora et al., 2005b), y que las recesiones económicas, el desempleo y los bajos ingresos se asocian con problemas de salud mental, principalmente en los relacionados con la ansiedad en las mujeres (Dagher, Chen \& Thomas, 2015). Asimismo, la educación inconclusa se relaciona con el consumo de sustancias, con la ansiedad y con los trastornos del estado de ánimo y de control de impulsos (Lee et al., 2009), mientras que, en cuanto al estado civil, estar separado, divorciado o viudo incrementa el riesgo de presentar trastornos mentales (Scott et al., 2009). Finalmente, otro aspecto por considerar es la violencia, ya que se ha encontrado que las mujeres que viven violencia conyugal presentan sintomatología de somatización, depresión, sensibilidad interpersonal y psicoticismo, lo que puede reflejarse en menor calidad de vida, enfermedades físicas y uso frecuente de los servicios de salud (Vieyra, Gurrola, Balcázar, Bonilla \& Virseda, 2009).

Sin embargo, a pesar de los avances en el torno a la salud y el reconocimiento de los determinantes sociales, también se presenta un descuido importante con respecto la atención prestada a la salud mental y a los factores que propician la aparición de síntomas incipientes, sobre todo cuando se trata de población comunitaria (no clínica), ya que esta ha sido utilizada principalmente como grupo de comparación de aquellas personas que presentan un trastorno mental. Al dejar de lado la población general (o abierta), se olvida la importancia que tienen los síntomas y los determinantes sociales en ella, pero su estudio serviría como fundamento para generar intervenciones de promoción, prevención y detección temprana, sobre todo en los países en vías de desarrollo, donde dichas estrategias son escasas (Berenzon, Lara, Robles \& Medina-Mora, 2013). Teniendo esto en cuenta, el objetivo del presente estudio fue identificar los factores psicosocioculturales que influyen en la presencia de síntomas psicopatológicos en población abierta, considerando a adultos de ambos sexos de la jurisdicción sanitaria Zumpango, Estado de México. 
172

\section{Método}

Se realizó un estudio cuantitativo, no experimental, transversal y de alcance explicativo, que, por medio de la elaboración de modelos predictivos, buscó mostrar los factores que inciden en los síntomas psicopatológicos de la población abierta (Herrnández, Fernández \& Baptista, 2014).

\section{Participantes}

Se trabajó con una muestra por conveniencia de participantes voluntarios, de población abierta, formada por 229 personas de ambos sexos (68.1\% mujeres y $31.9 \%$ hombres), adultos de 18 a 81 años $(X=35.15, D E=13.22)$, mayormente casados (40\%) y solteros (31\%), con estudios de nivel secundaria. Todos los participantes residían de la región de Zumpango del Estado de México, específicamente de los municipios de Apaxco, Huehuetoca, Hueyploxtla, Nextlalpan, Tequixquiac y Zumpango, los cuales, al pertenecer a la misma área geodemográfica, compartían recursos, actividades económicas y elementos socioculturales similares.

Esta región está ubicada al noreste del Estado de México, y es un Estado caracterizado por ser complejo, heterogéneo y el más poblado del país, con un proceso de modernización acelerada, desorganizada y desigual. De manera particular, la región en donde se aplicó este estudio se encuentra ubicada entre las zonas metropolitanas del Valle de México y del Estado de Hidalgo (Gobierno del Estado de México, COPLADEM, 2012), en donde se vive un proceso de desterritorialización, ya que el territorio es rebasado por cambios profundos debido a la fuerte presión sociodemográfica. Además, su población ha aumentado casi al doble en las últimas décadas, y ha transitado de lo rural a lo urbano, así como de lo agropecuario al comercio, con lo cual se genera una creciente demanda de recursos y servicios para cubrir las necesidades de la población (Sánchez, 2013).

Adicional a esto, la región cuenta con una población mayoritariamente urbana (75\%) y originaria ( $72 \%$ ), con un $28 \%$ de migración — principalmente de la Ciudad de México, hacia donde las personas se trasladan para trabajar y regresan solo a dormir-, además de que presenta niveles bajos de marginación, $10.4 \%$ de pobreza alimentaria y $33.4 \%$ pobreza patrimonial (Consejo Nacional de Evaluación de la Politica de Desarrollo Social, CONEVAL, 2016). Por último, aunque el índice de desarrollo humano en la región ha mejorado, ya que cuenta con tasas de mortalidad menor a la estatal, una esperanza de vida de 76.81 años, cobertura de atención primaria $(76 \%)$ y escolaridad promedio de nueve años; no obstante, las características señaladas se distribuyen de manera desigual e inequitativa, pues las zonas alejadas de los centros municipales se ven significativamente desfavorecidas (Gobierno del Estado de México [COPLADEM], 2012).

\section{Instrumentos}

Escala de Factores Asociados a la Salud. Se utilizó esta escala para recopilar, por una parte, los datos sociodemográficos (sexo, edad, escolaridad, estado civil) y, por otra, las factores psicosocioculturales asociados a la salud, teniendo en cuenta tanto los aspectos psicológicos como socioculturales y sanitarios relacionados con el proceso de salud-enfermedad, como lo son el estado de salud, la seguridad médica, la percepción de bienestar, los pensamientos y comportamientos problemáticos, la familia, la violencia, el desempleo, la pobreza, la inseguridad, la discriminación, la migración y el medio ambiente.

Específicamente, la escala está conformada por 26 reactivos que se responden mediante una escala tipo Likert de cinco puntos ("nunca", "casi nunca", "algunas veces", "casi siempre", "siempre"), en donde, en la calificación, a mayor puntaje, mayor presencia de la condición evaluada. Por último, es importante mencionar que este instrumento fue elaborado exprofeso para el proyecto de donde emanan los datos presentados; que la sección dedicada a los datos sociodemográficos ha sido utilizada previamente (Ornelas \& Ruiz, 2017); y que la sección de factores psicosocioculturales obtuvo para esta muestra una varianza explicada del $52 \%$ y una confiabilidad suficiente — con un alfa de .74-.

Inventario de síntomas (SCL-90-R). Esta lista de chequeo, elaborada por Derogatis (2002), es un instrumento de autorreporte que evalúa la presencia de síntomas psicopatológicos en la población evaluada. Específicamente, se trabajó con la versión validada en México por Cruz et al. (2005), conformada por 90 reactivos y una escala Likert de cinco opciones de respuesta ("nada", "muy poco", "poco", "bastante" y "mucho"), en la que el participante indica aquellos síntomas que le han preocupado o molestado durante los últimos siete días.

Para los resultados, a mayor puntuación, mayor sintomatología. Se utilizaron tanto la puntación total, categorizada como índice de severidad global (ISG), como las puntuaciones de las dimensiones (somatización, obsesiones y compulsiones, sensibilidad interpersonal, depresión, ansiedad, hostilidad, ansiedad fóbica, ideación paranoide, y psicoticismo). En la validación de Cruz et al. (2005), el instrumento reportó propiedades psicométricas nacionales e internacionales adecuadas (entre $\alpha=.60$ y $\alpha=.85$ ), y en 
el presente estudio se confirmó su validez — con un $64 \%$ de varianza explicada - y su confiabilidad — con un alfa de .98 -

\section{Procedimiento}

Una vez aprobado y registrado el proyecto ante las autoridades institucionales, se procedió a la selección de los aplicadores capacitados, quienes eran provenientes de las mismas zonas de recolección de datos, aspecto que facilitó el contacto con la comunidad. Posteriormente, se prosiguió con un periodo de familiarización para hacer visibles a los aplicadores ante los pobladores, y así, además de generar confianza, brindar seguridad para el equipo de investigación y facilitar la recolección de datos.

Después de esto, los aplicadores abordaron a los participantes en espacios públicos —como hospitales, iglesias, mercados, escuelas, etc. - y privados - como domicilios, comercios, etc.- Para esto, en primer lugar, los investigadores se identificaron, explicaron el objetivo de la investigación, y solicitaron su participación voluntaria; después, los participantes que aceptaron participar firmaron el consentimiento informado, el cual se realizó según la Declaración de Helsinki y los lineamientos éticos de la APA; acto seguido, los participantes contestaron los cuestionarios de manera individual; y, para finalizar, se verificó el llenado completo de los instrumentos y se agradeció por la participación.

\section{Análisis de datos}

Inicialmente, se realizó el análisis descriptivo $(M, D E)$; luego, se utilizó la prueba de normalidad KolmogorovSmirnov-Lilliefors y la prueba Levene para verificar la homogeneidad de varianza; y después se llevó a cabo un análisis comparativo entre mujeres y hombres ( $t$ de Student) - debido a que se encontraron diferencias significativas por sexo $(\mathrm{p} \leq .05)$, el resto de los análisis se realizaron de manera separada para mujeres y hombres-. Posteriormente, se elaboraron correlaciones bivariadas entre cada uno de los síntomas y los factores evaluados; y, por último, se utilizó la regresión lineal multivariada, paso a paso, para generar modelos predictivos para cada sexo. Durante todo el proceso de análisis de datos se utilizó el programa SPSS, versión 22.

\section{Resultados}

En esta sección se presentan los resultados del estudio. Se inicia con los datos demográficos y de salud, seguidos por la comparación entre los síntomas psicopatológicos y los factores psicosocioculturales entre sexos, así como la relación entre dichos síntomas y factores; y, por último, la elaboración de los modelos predictivos para las mujeres y para los hombres.

En cuanto a los datos demográficos, no se encontraron diferencias con respecto a la edad ni en el estado civil, pero sí en la escolaridad, siendo mayor el nivel en hombres que en mujeres $\left(X^{2}=18.20\right.$, sig. $\left.=.003\right)$. Con respecto a la salud, predominaron los usuarios del Seguro Popular $(31.0 \%)$, seguidos por aquellos que no tienen este tipo de servicios $(29.3 \%)$ y por los derechohabientes de seguro laboral (IMSS, $25 \%$ ); además, el $17.9 \%$ indicó que en el momento de la evaluación se encontraba enfermo, el 14.0\% recibía tratamiento, y el $18.82 \%$ tomaba medicamentos. En estos datos, las mujeres reportaron porcentajes más altos que los hombres, aunque de forma no significativa.

Con respecto a los problemas de salud mental, una vez que se comprobó la normalidad de las variables y la homogeneidad de varianzas —respetando la significancia de .05-, se encontraron — por medio de la t de Studentalgunas diferencias de género importantes, ya que las mujeres presentaron mayor sintomatología psicopatológica que los hombres: el $15.4 \%$ de las mujeres obtuvo altas puntuaciones en el SCL 90-R, en comparación con el $5.5 \%$ de los hombres, con diferencias significativas en siete de las nueve dimensiones evaluadas. Específicamente, en el caso de las mujeres, las mayores puntuaciones se encontraron en ideación paranoide, obsesivo-compulsivo, depresión y somatización; mientras que, en el caso de los hombres, dichas puntuaciones se ubicaron en obsesivo-compulsivo, sensibilidad interpersonal, somatización y hostilidad (véase Tabla 1).

Tabla 1.

Comparación de síntomas psicopatológicos entre mujeres y hombres

\begin{tabular}{lccc}
\hline \multicolumn{1}{c}{ Síntomas } & Mujeres & Hombres & \\
& $\mathrm{n}=156$ & $\mathrm{n}=73$ & $t$ de \\
& $M(D E)$ & $M(D E)$ & Student \\
\hline Somatización & $.74(.69)$ & $.40(.48)$ & $4.35 * * *$ \\
Obsesivo-compulsivo & $.75(.74)$ & $.61(.64)$ & 1.48 \\
Sensibilidad interpersonal & $.67(.68)$ & $.45(.50)$ & $2.76^{* *}$ \\
Depresión & $.74(.78)$ & $.37(.53)$ & $4.20^{* * *}$ \\
Ansiedad & $.64(.72)$ & $.39(.53)$ & $2.71^{* *}$ \\
Hostilidad & $.54(.59)$ & $.40(.47)$ & 2.04 \\
Ansiedad fóbica & $.51(.65)$ & $.25(.46)$ & $3.47 * *$ \\
Ideación paranoide & $.76(.77)$ & $.50(.65)$ & $2.62^{*}$ \\
Psicoticismo & $.45(.59)$ & $.25(.42)$ & $2.84 * *$ \\
Índice de severidad global & $.65(.60)$ & $.48(.57)$ & $3.43 * * *$ \\
\hline
\end{tabular}

Nota. $M=$ media, $D E=$ desviación estándar. ${ }^{*} \mathrm{p}<.05,{ }^{*} \mathrm{p}<.01$, $* * * \mathrm{p}<.001$. Fuente: elaboración propia. 
En cuanto a los factores psicosocioculturales asociados a la salud, se encontraron algunas diferencias entre mujeres y hombres (en la Tabla 2 se presenta el resumen de estas comparaciones). En general, las mujeres indicaron mayor frecuencia de problemas emocionales, desintegración familiar, bajos ingresos/pobreza y mayor percepción de inseguridad en la comunidad, mientras que los hombres presentaron mayor actividad física, bienestar y acciones para la comunidad.

Siguiendo las recomendaciones de Grande, Newmeyer, Underwood y Williams (2014), se utilizó la puntuación de la sintomatología global (ISG), como medida estable que concentra los síntomas psicopatológicos, y posteriormente se realizaron las correlaciones entre el total de síntomas psicopatológicos (ISG) y los factores psicosocioculturales. En la Tabla 3 se resumen estas correlaciones, con un $r \geq .30$.

Se encontraron relaciones significativas para ambos sexos entre los factores psicosocioculturales y la psicopatología total (ISG), aunque el sexo femenino presentó mayor número de correlaciones y con intensidad más elevada, lo que, considerando el tamaño de la muestra, indica que son relaciones importantes. Esta relación entre variables muestra que existen diversos aspectos psicológicos, familiares y sociales asociados a la sintomatología, además que en las mujeres lo individual y familiar tiene mayor relación que en los hombres.

Los factores psicosocioculturales que obtuvieron correlaciones significativas se utilizaron como variables

Tabla 2.

Comparación de factores psicosocioculturales entre mujeres y hombres

\begin{tabular}{lccc}
\hline \multicolumn{1}{c}{ Variable } & Mujeres $(\mathrm{n}=156)$ & Hombres $(\mathrm{n}=73)$ & $\mathrm{t}$ de \\
& $M(D E)$ & $M(D E)$ & Student \\
\hline Actividad física & $1.58(1.2)$ & $2.33(1.1)$ & $4.27 * * *$ \\
Bienestar & $2.80(1.0)$ & $3.19(0.9)$ & $2.66 * *$ \\
Acciones por la comunidad & $1.43(1.1)$ & $1.83(1.4)$ & $2.07 *$ \\
Problemas emocionales & $1.11(1.2)$ & $0.79(0.99)$ & $2.09 *$ \\
Desintegración familiar & $0.89(1.4)$ & $0.50(1.1)$ & $2.12 *$ \\
Pobreza & $1.34(1.1)$ & $0.91(0.85)$ & $3.18 * *$ \\
Inseguridad en la comunidad & $2.77(1.2)$ & $2.33(1.2)$ & $2.48 * *$ \\
\hline
\end{tabular}

Nota. $\mathrm{M}=$ media, $\mathrm{DE}=$ desviación estándar. ${ }^{*} \mathrm{p}<.05,{ }^{* *} \mathrm{p}<.01,{ }^{* * *} \mathrm{p}<.001$. Fuente: elaboración propia.

Tabla 3.

Relación entre los factores psicosocioculturales y los síntomas psicopatológicos

\begin{tabular}{|c|c|c|}
\hline \multirow{2}{*}{ Variable } & \multicolumn{2}{|c|}{ Índice de severidad global } \\
\hline & Mujeres $(n=156)$ & Hombres $(n=73)$ \\
\hline $\mathrm{Su}$ forma de pensar le genera problemas & $.48 * * *$ & $.44 * * *$ \\
\hline Problemas emocionales & $.47 * * *$ & $.34 * *$ \\
\hline $\mathrm{Su}$ forma de actuar le genera problemas & $.41 * * *$ & $.41 * * *$ \\
\hline Confianza en sus capacidades & $-.41 * * *$ & - \\
\hline Sentimiento de productividad & $-.38 * * *$ & $-.39 * *$ \\
\hline Enfrenta y resuelve sus problemas & $-.38 * * *$ & - \\
\hline Bienestar & $-.37 * * *$ & $-.41 * * *$ \\
\hline Alimentación balanceada & $-.34 * * *$ & - \\
\hline Violencia intrafamiliar & $.47 * * *$ & $.45 * * *$ \\
\hline Conflictos familiares & $.44 * * *$ & $.38 * *$ \\
\hline Comunicación familiar & $-.43 * * *$ & - \\
\hline Afecto y aceptación familiar & $-.43 * * *$ & - \\
\hline Apoyo familiar & $-.37 * * *$ & - \\
\hline Desintegración familiar & $.31 * * *$ & $.38 * *$ \\
\hline Discriminación de género en la comunidad & $.38 * * *$ & - \\
\hline Pobreza/bajos ingresos & - & $.38^{*}$ \\
\hline Acceso a servicios de salud & - & $-.37 *$ \\
\hline Relaciones interpersonales agradables & - & $-.34 * *$ \\
\hline
\end{tabular}

Nota. $r=$ correlación de Pearson. ${ }^{*} \mathrm{p}<.05,{ }^{*} \mathrm{p}<.01, * * * \mathrm{p}<.001$. Fuente: elaboración propia. 
Tabla 4.

Modelos predictivos de los factores psicosocioculturales sobre los sintomas psicopatológicos en mujeres y hombres

\begin{tabular}{|c|c|c|c|c|c|c|}
\hline \multirow{2}{*}{ Variables predictoras } & \multicolumn{6}{|c|}{ Índice de severidad global } \\
\hline & $B$ & $\mathrm{~T}$ & Sig. & $\mathrm{R}^{2}$ & $\mathrm{~F}$ & Sig. \\
\hline Mujeres $(\mathrm{n}=156)$ & & & & .48 & 35.60 & .000 \\
\hline Pensamientos que generan problemas & .29 & 4.61 & .000 & & & \\
\hline Violencia intrafamiliar & .31 & 4.90 & .000 & & & \\
\hline Confianza en sus capacidades & -.30 & -5.05 & .000 & & & \\
\hline Discriminación de género & .21 & 3.36 & .001 & & & \\
\hline Hombres $(\mathrm{n}=73)$ & & & & .38 & 14.99 & .000 \\
\hline Violencia intrafamiliar & .40 & 4.14 & .000 & & & \\
\hline Bienestar & -.29 & -2.93 & .005 & & & \\
\hline Pobreza/bajos ingresos & .28 & 2.91 & .005 & & & \\
\hline
\end{tabular}

Nota. $B=$ Beta, coeficiente de regresión estandarizado, Valor $\mathrm{T}\left(\mathrm{T}\right.$, sig.), $\mathrm{R}^{2}$ = coeficiente de determinación ajustado, ANOVA (F, sig.). Fuente: elaboración propia.

predictoras sobre los síntomas psicopatológicos para generar los modelos explicativos mediante regresiones múltiples, paso a paso, con lo que se procedió a elegir el modelo que presentara el mejor ajuste. Así, el modelo femenino identificó cuatro variables que explicaban el $48 \%$ de la varianza; en tanto que el modelo masculino estuvo compuesto por tres variables predictoras, con el $38 \%$ de la varianza, una de ellas con efecto inverso; con lo que se concluye que un menor bienestar predice la sintomatología. Aunque los dos modelos son significativos, el modelo femenino presentó mejor calidad de ajuste y valor predictivo que el masculino (véase Tabla 4); las variables contenidas en ambos modelos son diferentes, a excepción de violencia intrafamiliar, que influye en ambos sexos como el predictor más fuerte.

\section{Discusión}

Con el fin de identificar los diferentes factores que inciden sobre los problemas de salud mental en personas adultas de población abierta, el presente estudio relacionó los factores psicosocioculturales con los síntomas psicopatológicos en una muestra de 229 participantes. Para el análisis, se inició con la comparación de las variables de interés entre mujeres y hombres, $y$, dado que se encontraron diferencias significativas, se mantuvo la distinción por sexo a lo largo del estudio, hasta la generación de los modelos explicativos.

En general, los resultados sobre los síntomas psicopatológicos en el presente estudio tienden a ser mayores que los reportados en otros estudios, principalmente para las mujeres (Cruz et al., 2005; Lara, Espinosa, Cárdenas, Fócil \& Cavazos, 2005), aspecto que representa un riesgo aumentado, como suele darse en zonas urbanizadas donde la modernización no es planificada y las zonas rurales se ven despojadas de sus recursos (OPS, 2018a). Es posible que esto responda al proceso de urbanización acelerado que viven los participantes de este estudio, ya que ha sido poco planeado, es desorganizado, y hace que los habitantes se encuentren en procesos de adaptación constantes — con estresores propios de las zonas urbanas, como los problemas en las zonas dormitorio-, ya que hacen falta servicios y oportunidades laborales, razón por la cual las personas se encuentran en la búsqueda permanente de alternativas que conllevan esfuerzos redoblados y largas distancias para cubrir sus necesidades. Por supuesto, estos datos están supeditados a la muestra utilizada (intencional y voluntaria); por lo que en futuras investigaciones se requieren muestras representativas que cubran diferentes grados de urbanización.

Asimismo, las mujeres del presente estudio presentaron mayor sintomatología que los hombres en la mayoría de las dimensiones del SCL 90-R, lo cual confirma que ellas presentan menor salud mental percibida (Gagne, Vasiliadis \& Préville, 2014). Esta tendencia concuerda con los estudios que reportan que las mujeres presentaron mayor psicopatología en casi todas las dimensiones evaluadas (Alvarado et al., 2012; Ruiz et al., 2018); pero se distancia de aquellos autores que refieren escasas o nulas diferencias (Cruz et al., 2005; Kröninger \& Grevenstein, 2013).

En el presente estudio las mayores diferencias ocurrieron en las dimensiones de somatización, depresión, ansiedad fóbica y sintomatología total, las cuales aparecen de manera consistente en la literatura, especialmente la depresión y la ansiedad (Luppa et al., 2012). Incluso, se ha reportado que las mujeres presentan ligeramente más recaídas que los hombres (Bertschy, Velten \& Weibel, 2016). Estos hallazgos sobre la sintomatología podrían interpretarse como característica individual, pero, si se dejan en ese plano, se correría el riesgo de presentar una visión individualista - $\mathrm{e}$ 
176

incluso psicologicista - al dar a entender que esos síntomas serían características individuales aisladas. En consecuencia, fue necesario ampliar el panorama para contemplar otros niveles del ecosistema que dieran cuenta de los elementos contextuales que inciden en la salud mental.

Así, al comparar los factores psicosocioculturales asociados a la salud en mujeres y hombres, se encontró que las mujeres se perciben con menos bienestar y participación en acciones comunitarias, además de que presentan más problemas emocionales, desintegración familiar, pobreza e inseguridad en la comunidad que los hombres. Algunos de los aspectos encontrados, como bajos ingresos, falta de educación e inseguridad dentro de su medio ambiente, han sido citados con anterioridad como influyentes en la inequidad sanitaria (OMS, 2009; OPS, 2018a) y en la presencia de trastornos mentales (OMS, 2013); pero, en el presente estudio, que se realizó en una región que ha transitado recientemente de lo rural a lo urbano, esto se manifiesta principalmente en las mujeres.

Por otra parte, al estudiar la relación entre las variables de interés mediante análisis correlacionales bivariados, se confirmó la asociación entre los factores psicológicos, familiares, socioculturales y la presencia de recientes síntomas psicopatológicos, como se ha mostrado previamente en afectaciones a lo largo de la vida (Benjet et al., 2010). Específicamente, las variables que mostraron relaciones más estrechas con la sintomatología total en ambos sexos fueron los factores psicológicos - como percibir que su forma de pensar y actuar les genera problemas, afectaciones emocionales - y los factores familiares - como la violencia intrafamiliar, los conflictos y la desintegración familiar-. Cabe señalar que las asociaciones fueron de mayor magnitud para las mujeres, y que en los factores socioculturales no hubo coincidencias.

En cambio, se presentaron otras relaciones con la sintomatología que solo se establecieron para alguno de los sexos, como que las mujeres mostraron menor confianza en sus capacidades, capacidad afrontamiento, y comunicación, afecto y apoyo familiar; mientras que en los hombres fue común la pobreza y menos servicios y relaciones interpersonales. Esto daría cuenta de que los participantes de población abierta logran identificar que los aspectos cognitivo-conductuales, emocionales y familiares les generan problemas; mientras que la disminución de aspectos positivos — como sentirse bien y útiles - les genera malestar. De esta manera, los resultados obtenidos sobre los síntomas y factores individuales darían cuenta del ontosistema, los factores familiares del microsistema y los factores socioculturales del macrosistema, con lo cual se logra ver el complejo entretejido de relaciones con la psicopatología. De esta forma, se deja claro que los factores asociados al malestar psicológico atraviesan los distintos niveles ecosistémicos.

Con esto último se confirman las múltiples relaciones entre los factores psicológicos y los síntomas psicopatológicos, algunas coincidentes, y otras dependen del sexo de los participantes. Estas coincidencias señalan que la presencia de problemas cognitivos, conductuales y emocionales, así como sentirse menos productivo y con menor bienestar, se asocian con los síntomas psicopatológicos, con lo cual destaca la relevancia del bienestar psicológico sobre la salud mental (Alvarado et al., 2013). En particular, en las mujeres la falta de confianza en sus capacidades y el escaso afrontamiento ante sus problemas se relacionaron con la sintomatología total, variables que no resultaron significativas para los hombres. Esto concuerda parcialmente con otros estudios que señalan la relación negativa entre la autoeficacia y la sintomatología (Gull, 2016; Kröninger $\&$ Grevenstein, 2013), aunque en el presente trabajo solo aplica para las mujeres.

En cuanto al afrontamiento, otros estudios han detallado que algunas estrategias, como culparse a sí mismo o la despersonalización, se relacionan con problemas de salud mental — como la depresión- y con algunas diferencias por género —como que las mujeres utilizan afrontamiento activo, apoyo emocional e instrumental, solución de problemas, preocupación y espiritualidad, mientras que los hombres utilizan el consumo de sustancias e ignoran el problema (Fantin, Florentino \& Correché, 2005; Ito \& Matsushima, 2017)—. El presente estudio es limitado en cuanto a la aproximación hacia las estrategias de afrontamiento, por lo que se requiere profundizar mediante el uso de instrumentos evalúen esta variable.

Por otra parte, se encontraron relaciones entre los factores familiares y la sintomatología, algunas coincidentes para ambos sexos, pero de mayor magnitud en las mujeres. Por ejemplo, se encontró que, a mayor violencia intrafamiliar, conflictos y desintegración familiar, más aumentan los problemas de salud mental en ambos sexos. Esto coincide con estudios que señalan a los problemas familiares como principal estresor para la presencia de problemas de salud mental, en donde se detalla una amplia gama de aspectos, como las malas relaciones, la infidelidad, la violencia, el abandono, el maltrato y los problemas con los hijos y la pareja, lo cual es percibido tanto por los usuarios que solicitan atención como por los psicólogos que atienden en el primer nivel de atención (Ornelas \& Ruíz, 2016; Saavedra \& Uchofen, 2016).

Es importante destacar que en el caso de las mujeres también se encontraron algunas relaciones inversas que indican que, a menor comunicación, afecto y apoyo familiar, más problemas de salud mental; aunque estas variables no 
resultaron relevantes para el sexo masculino, posiblemente debido a que la figura femenina está más asociada con el apoyo y la comunicación afectiva (Garcés \& Palacio, 2010).

Si bien la familia es importante para ambos sexos, no lo es de la misma manera y no todos los aspectos adquieren la misma importancia según el sexo: por ejemplo, para las mujeres existe malestar psicológico cuando aumentan los problemas familiares y cuando disminuyen los aspectos positivos del funcionamiento familiar; pero en los hombres solo se establece dicha relación cuando aumenta lo problemático. Posiblemente, la salud mental de las mujeres está más vinculada con sus relaciones familiares, ya que de manera histórica el hogar y el espacio doméstico han sido asignado a las mujeres como parte del rol tradicional femenino - en el que se les identifica como procreadoras, cuidadoras de los otros, encargadas de lo afectivo-, en donde la figura materna adquiere primacía como figura abnegada y sacrificada; en cambio, en los hombres se niega el reconocimiento de sus afectos y de su expresión emocional, además de que se favorece la independencia y se reconoce lo público como su espacio de desarrollo (Rabell, 2009). Quizá lo encontrado tenga que ver con la ternura negada y la búsqueda de apoyo como sinónimo de debilidad (Espinosa et al., 2015), significados inculcados a los hombres en la cultura patriarcal.

En cuanto los factores socioculturales, se presentaron más relaciones con los síntomas psicopatológicos en hombres que en mujeres, y las variables asociadas fueron diferentes para ambos sexos. En las mujeres, la discriminación de género fue la única variable sociocultural relacionada con la sintomatología total - con lo que se reitera que la inequidad, desigualdad y subordinación afectan más a las mujeres (Ruiz, 2011) - , mientras que, para los hombres, la percepción de pobreza, menos relaciones sociales y la falta de acceso a los servicios de salud se relacionaron con la presencia de síntomas psicopatológicos —con lo que se demuestra la relevancia del aspecto económico y el ámbito público en la salud mental masculina- Al respecto, la literatura previa señala que la distribución desigual de ingresos y servicios impacta sobre la salud y la atención recibida por la población general, además de que favorece la presencia de problemas de salud mental (Medina-Mora et al., 2005b). En el presente estudio, estos factores de exclusión social fueron resentidos por los hombres, pero no en las mujeres.

Como parte final del análisis, en este trabajo se elaboraron los modelos que permitieron integrar los factores psicológicos, familiares y socioculturales con mayor valor predictivo sobre los síntomas psicopatológicos, con lo que se demostró que dichos factores siguen diferentes vías de influencia, dependiendo del sexo de los participantes. La única variable coincidente en ambos modelos fue la violencia intrafamiliar, aspecto que resalta el impacto que tiene esta variable sobre la salud mental de la población. Al respecto, en la literatura se ha enfatizado que la violencia conyugal en las mujeres se asocia con la psicopatología (Vieyra et al., 2009), así como con el malestar psicológico y las creencias que acompañan los actos violentos que cometen los hombres (Bolaños, 2014). De manera específica, se ha documentado que la violencia de pareja, expresada mediante amenazas y maltrato físico, afecta la salud física y mental, así como la calidad de sueño en hombres y mujeres (Lalley et al., 2017). Con esto se da cuenta de que la violencia intrafamiliar afecta a todos los implicados en el sistema familiar.

Específicamente, en el modelo femenino las variables predictoras de síntomas psicopatológicos fueron la violencia intrafamiliar, la falta de confianza en sus capacidades, los pensamientos problemáticos y la discriminación de género, con lo que se demuestra que a las mujeres les afectan estas características individuales y familiares —así como, la discriminación de género en su comunidad-, pero ello no ocurre con los hombres. Esto coincide parcialmente con lo propuesto por Kucharska (2017), en donde se presenta la interrelación entre las variables contextuales y los problemas de salud mental, mediados por variables individuales.

En cambio, en el modelo masculino las variables predictoras fueron la violencia intrafamiliar, el bienestar disminuido y la pobreza. Es importante recordar que en este estudio los hombres presentaron mayor bienestar y menor pobreza que las mujeres, pero, a pesar de estar mejor que las mujeres, estas variables adquieren tal importancia en los hombres que predicen la psicopatología global. Esto podría estar asociado a la búsqueda de satisfacción individual, el impacto de la violencia ejercida y la relevancia del aspecto económico para los hombres, lo que podría coincidir con los malestares asociados al rol masculino tradicional (Fleiz, Ito, Medina-Mora \& Ramos, 2008).

En consecuencia, se considera que estas aportaciones refuerzan el argumento de que las manifestaciones psicopatológicas requieren ser entendidas desde posturas teóricas que vayan más allá de lo individual y lo biomédico, porque los síntomas no ocurren de manera aislada y los factores encontrados para mujeres y hombres se entretejen en complejas interrelaciones. Esto muestra la necesidad de utilizar modelos psicosociales y ecosistémicos que permitan dar cuenta de los múltiples factores involucrados en los síntomas incipientes que ya se encuentran presentes en la población abierta, pero que pasan por alto ante el común de las personas y los trabajadores de la salud de primer contacto. Estos síntomas deberían ser detectados 
oportunamente, sin esperar a que se conviertan en casos clínicos con las complicaciones y costos que ello conlleva.

Finalmente, se advierte que los resultados del presente trabajo deberán contemplarse desde las limitaciones teóricas y metodológicas de un trabajo cuantitativo, transversal y basado en autorreportes, por lo que en el futuro se recomienda trabajar con muestras representativas y diversos grupos de comparación en cuanto a la sintomatología (población abierta y clínica) y la urbanización (rural, semiurbana y urbana); así como profundizar en el contenido desde las perspectivas psicosociales y metodologías participativas.

Ampliar el panorama teórico y metodológico enriquecería la base para la elaboración de programas de promoción de la salud mental y prevención de los principales síntomas encontrados; con esto se podría trascender la visión psicologizante y, por tanto, entender los presentes resultados con una mirada más amplia que permita explorar y reconocer los determinantes sociales y las capacidades de la comunidad para generar mejores condiciones asociadas a la salud. Al respecto, se reconoce la relevancia los estudios etnográficos y las propuestas generadas desde la perspectiva de la investigación acción participativa (IAP) para trabajar aspectos socioculturales (Rodríguez-Mancilla \& Boada, 2016).

En síntesis, los resultados de la presente investigación pueden resultar útiles para mostrar que, en una sociedad con una modernización desorganizada como la nuestra, la violencia, la inequidad de género y la desigualdad económica generan malestar psicopatológico tanto en mujeres como en hombres. De tal forma, los problemas de salud mental se encuentran vinculados a la desigualdad social y enfatizan las diferencias de género y económicas, lo cual se convierte en la normatividad social que delinea el desarrollo de los individuos, quienes a su vez replican estos modelos en su vida cotidiana, aunque al mismo tiempo les genere malestar.

\section{Referencias}

Alvarado, B. G., Sandín, B., Valdez-Medina, J. L., GonzálezArratia, N., \& Rivera S. (2012). Análisis factorial confirmatorio del Cuestionario SA-45 en una muestra mexicana. Anales de Psicología, 28(2), 426-433. doi: 10.6018/ analesps.28.2.148851

Alvarado, R., Barquero, S. P., Garita, M. P., Hernández, C. M., Leandro, R. M., ... Sotela, S. G. (2013). Estado del arte de la investigación en salud mental en Costa Rica. Universidad de Costa Rica.

Benjet, C., Borges, G., \& Medina-Mora, M. E. (2010). Chronic childhood adversity and onset of psychopathology during three life stages: Childhood, adolescence and adulthood. Journal of Psychiatric Research, 44(11), 732-740. doi: 10.1016/j.jpsychires.2010.01.004
Berenzon, S., Lara, M. A., Robles, R., \& Medina-Mora, M. E. (2013). Depresión: estado del conocimiento y la necesidad de políticas públicas y planes de acción en México. Salud Pública de México, 55(1), 74-80. doi: 10.1590/ S0036-36342013000100011

Bertschy, G., Velten, M., \& Weibel, S. (2016). Major depression: does gender influence the risk of recurrence? A systematic review. The European Journal of Psychiatry, 30(1), 7-27. Recuperado de http://scielo.isciii.es/scielo. php?script=sci_arttext\&pid=S0213-61632016000100001

Bolaños, C. F. (2014). Psychological distress socially determined and expressive abuse in men. Salud Mental, 37(4), 321-327. doi: 10.17711/SM.0185-3325.2014.037

Breslau, J., Aguilar-Gaxiola, S., Borges, G., Castilla-Puentes, R. C., Kendler, K. S., ... Kessler, R. C. (2007). Mental disorders among English-speaking Mexican immigrants to the US compared to a national sample of Mexicans. Psychiatry Research, 15(1-2), 115-122. doi: 10.1016/j. psychres.2006.09.011

Consejo Nacional de Evaluación de la Política de Desarrollo Social [CONEVAL]. (2016). Índice de rezago social 2015 a nivel nacional, estatal y municipal. México: CONEVAL. Recuperado de

Cruz, F. C., López, B. L., Blas, G. C., González, M. L., \& Chávez, B. R. (2005). Datos sobre la validez y confiabilidad de la Symptom Check List 90 (SCL 90) en una muestra de sujetos mexicanos. Salud Mental, 28(1), 72-81. Recuperado de http://www.revistasaludmental.mx/index. php/salud_mental/article/view/1037

Dagher, R. K., Chen, J., \& Thomas, S. B. (2015). Gender differences in mental health outcomes before, during, and after the Great Recession. Plos ONE, 10(5). doi: 10.1371/jour nal.pone.0124103

Derogatis, L. R. (2002). SCL-90-R Symptom Checklist-90-R. Cuestionario de 90 síntomas (Adaptación Española por González de Rivera, J. L., de las Cuevas, C., Rodríguez A., \& Rodríguez P. F.). España: Pearson.

Espinosa, M. M., Orozco, R. L., \& Ybarra, S. J. (2015). Síntomas de ansiedad y depresión y factores psicosociales en hombres que solicitan atención de salud en el primer nivel. Salud Mental, 8(3), 201-208. doi: http://dx.doi. org/10.17711/SM.0185-3325.2015.028

Fantin, M. B., Florentino, M. T., \& Correché, M. S. (2005). Estilos de personalidad y estrategias de afrontamiento en adolescentes de una escuela privada de la ciudad de San Luis. Fundamentos en humanidades, 4(11), 163-180. Recuperado de http://www.redalyc.org/articulo.oa?id=18411609

Fleiz, B. C., Ito, M. E., Medina-Mora, I. M. E., \& Ramos, L. L. (2008). Los malestares masculinos: Narraciones de un grupo de varones adultos de la Ciudad de México. Salud Mental, 31(5), 381-390. Recuperado de http:// www.scielo.org.mx/scielo.php?script $=$ sci_abstract\&pid $=\mathrm{S} 0185-33252008000500006 \& \operatorname{lng}=\mathrm{es} \& \mathrm{nrm}=\mathrm{iso}$ 
Gagne, S., Vasiliadis, H. M., \& Préville, M. (2014). Gender differences in general and specialty outpatient mental health service use for depression. BMC Psychiatry, 14(135), 135-145. Recuperado de http://www.biomedcentral. com/1471-244X/14/135

Garcés, P. M., \& Palacio, S. J. (2010). La comunicación familiar en asentamientos subnormales de Montería. Psicología desde el Caribe, 25(1), 1-29. Recuperado de https://www. redalyc. org/articulo.oa?id=21315106002

Gobierno del Estado de México. (2012). Región XVI Zumpango. Programa regional 2012-2017. México: Comité de Planeación para el Desarrollo del Estado de México (COPLADEM). Recuperado de https://transparen ciafiscal.edomex.gob.mx/sites/transparenciafiscal.edomex. gob.mx/files/files/pdf/marco-programatico-presupuestal/ REGION_XVI.pdf

Grande, T. L., Newmeyer, M. D., Underwood, L. A., \& Williams, C.R. (2014). Path analysis of the SCL 90-R: Exploring use in outpatient assessment. Measurement and Evaluation in Counseling and Development, 47(4), 271290. doi: $10.1177 / 0748175614538061$

Gull, M. (2016). Self-Efficacy and mental health among professional students: A correlational study. International Journal of Modern Social Sciences, 5(1), 42-51. Recuperado de https://www.researchgate.net/publication/299535450 Self-Efficacy_and_Mental_Health_among_Professional_ Students_A_Correlational_Study

Hernández, R., Fernández, C., \& Baptista, P. (2014). Metodología de la investigación (6. ${ }^{\mathrm{a}}$ ed.). México: Mc Graw Hill Education.

Ito, M., \& Matsushima, E. (2017). Presentation of coping strategies associated with physical and mental health during health check-ups. Community Mental Health Journal, 53(3), 297-305. doi: 10.1007/s10597-016-0048-9

Kisely, S., Alichniewicz, K. K., Black, E. B., Siskind, D., Spurling, G., \& Toombs, M. (2017). The prevalence of depression and anxiety disorders in indigenous people of the Americas: A systematic review and meta-analysis. Journal of Psychiatric Research, 84(19), 137-152. doi: https://doi. org/10.1016/j.jpsychires.2016.09.032

Kohn, R., Levav, I, Caldas, A. J., Vicente, B., Andrade, L., ... Saraceno, B. (2005). Los trastornos mentales en América Latina y el Caribe: asunto prioritario para la salud pública. Revista Panamericana de Salud Pública, 18(4/5), 229-240. Recuperado de http://publications.paho.org/spanish/01_ ARTI_Kohn.pdf

Kröninger, J. H., \& Grevenstein, D. (2013). Development of salutogenetic factors in mental health-Antonovsky's sense of coherence and Bandura's self-efficacy related to Derogatis'symptom check list (SCL-90-R). Health and Quality life Outcomes, 11(1), 1-9. doi: https://doi. org/10.1186/1477-7525-11-80

Kucharska, J. (2017). Cumulative trauma, gender discrimination and mental health in women: mediating role of self-esteem. Journal of Mental Health, 27(5), 416-423. doi: 10.1080/09638237.2017.1417548

Lalley, C. L., Segal, A., Perlis, M., Nowakowski, S., Tal, J., \& Gradner, M. (2017). Sleep disturbance partially mediates the relationship between intimate, partner violence and physical/mental health in women and men. Journal of Interpersonal Violence, 32(16), 2471-2495. doi: $10.1177 / 0886260515592651$

Lara, C., Espinosa, I., Cárdenas. M., Fócil, M., \& Cavazos, J. (2005). Confiabilidad y validez de la SCL-90 en la evaluación de psicopatología en mujeres. Salud Mental, 28(3), 4250. Recuperado de http://revistasaludmental.mx/index.php/ salud_mental/article/view/1052

Lee, S., Tsang, A., Breslau, J. Aguilar-Gaxiola, S., Angermeyer, M., ... Kessler, R. C. (2009). Mental disorders and termination of education in high-income and middle-income countries: epidemiological study. British Journal of Psychiatry, 194(5), 411-417. doi: 10.1192/bjp.bp.108.054841

Luppa, M., Sirkorski, C., Ehreke, L., Konnopka, A., Wiese, B., ... Riedel-Heller, S. (2012). Age and gender specific prevalence of depression in latest-life: Systematic review and meta-analysis. Journal of Affective Disorders, 136(3), 212221. doi: 10.1016/j.jad.2010.11.033

Medina-Mora, M. E., Borges, G., Fleiz, C., Lara, C, Zambrano, R. J., \& Ramos, L. (2005a). Prevalencia de sucesos violentos y trastornos por estrés postraumático en población mexicana. Salud Pública de México, 47(1), 8-22. Recuperado de http://saludpublica.mx/index.php/spm/ article/view/6585/8122

Medina-Mora, M. E., Borges, G., Lara, C., Benjet, C., Blanco, J., ... Aguilar-Gaxiola, S. (2003). Prevalence of mental disorders and use of services: Results from the Mexican National Survey of Psychiatric Epidemiologic. Salud Mental, 26(4), 1-16. Recuperado de http://www.revistasalud mental.mx/index.php/salud_mental/article/view/956

Medina-Mora, M. E., Borges, G., Lara, C., Benjet, C., Blanco, J., ... Zambrano, J. (2005b). Prevalence, service use, and demographic correlates of 12-month DSM-IV psychiatric disorders in México: Results from the Mexican National Comorbidity Survey. Psychological Medicine, 35(2), 17731783. doi: 10.1017/S0033291705005672

Organización Mundial de la Salud [OMS]. (2003). Mental Health Context. Ginebra, Suiza: OMS.

Organización Mundial de la Salud [OMS]. (2009). Subsanar las desigualdades en una generación: alcanzar la equidad sanitaria actuando sobre los determinantes sociales de la salud: Informe final de la Comisión sobre Determinantes Sociales de la Salud. Argentina: Ediciones Journal.

Organización Mundial de la Salud [OMS]. (2013). Plan de acción sobre salud mental 2013-2020. Ginebra, Suiza: OMS.

Organización Panamericana de la Salud [OPS]. (2017). Salud en las Américas, edición 2017. Resumen: panorama regional y perfiles del pais. Washington, D. C.: OPS. 
180

Organización Panamericana de la Salud [OPS]. (2018a). Sociedades justas: equidad en la salud y la vida digna. Resumen ejecutivo del Informe de la Comisión de la Organización Panamericana de la Salud sobre equidad e desigualdades en salud en las Américas. Washington, D. C.: OPS.

Organización Panamericana de la Salud [OPS]. (2018b). La carga de los trastornos mentales en la Región de las Américas. Washington, D. C.: OPS.

Ornelas, R. A., \& Ruiz, M. A. O. (2016). Problemas de salud mental: asimetría entre las necesidades y los servicios brindados a la población general. Psicología Iberoamericana, 24(2), 63-73. Recuperado de https://www.redalyc.org/ar ticulo.oa?id=133949832008

Ornelas, R. A., \& Ruiz, M. A. O. (2017). Salud mental y calidad de vida: su relación en los grupos etarios. Psiencia. Revista Latinoamericana de Ciencia Psicológica, 9(2). doi: 105872/psiencia/9.2.21

Rabell, R. C. (2009). Tramas familiares en el México contemporáneo: Una perspectiva sociodemográfica. México: UNAM, Instituto de Investigaciones Sociales, El Colegio de México.

Rivera, L. A., Caballero, S. N., Pérez, S. I., \& Montero, L. L. (2013). SCL-90 R: distrés psicológico, género y conductas de riesgo. Universitas Psychologica, 12(1), 105-118.

Rodríguez, J. J. (2009). Salud mental en la comunidad. Washington, D. C.: OPS.

Rodríguez-Mancilla, M., \& Boada, M. J. (2016). Investigación acción participativa y renovación urbana en el barrio patrimonial la ronda de Quito. Revista Interamericana de Psicología, 50(1), 86-95. doi: 10.30849/rip/ijp.v50i1.94
Ruiz, M. A., González-Arratia, L. F. N. I., González, E. S., Aguilar, M. O., \& Torres, M. M. (2018). Relación entre síntomas psicopatológicos y calidad de vida en mujeres y hombres. Liberabit, 24(1), 97-113

Ruiz, M. M. (2011). La salud mental en México ¿Un problema de género? Revista Jurídica Jalisciense, 43, 93-112. Recuperado de http://148.202.18.157/sitios/publicacionesi te/pperiod/jurjal/jurjal01/93.pdf

Saavedra, J. E., \& Uchofen, H., V. (2016). Percepciones sobre la atención de salud en personas con problemas autoidentificados de salud mental en zonas rurales de Perú. Revista Peruana de Medicina Experimental y Salud Pública, 3(4), 785-793. doi: 10.17843/rpmesp.2016.334.2566

Sánchez, A. A. (2013). Actores locales y desarrollo de la región de Zumpango. En G. Alejandre, J. Pineda \& Y. Hernández (coord.), Región y desarrollo territorial. México entre lo urbano y lo rural (pp. 53-108). México. Parentalia ediciones.

Scott, K. M., Wells, J. E., Angermeyer, M., Brugha, T. S., Bromet, E., ... Kessler, R. C. (2009). Gender and the relationship between marital status and first onset of mood, anxiety and substance use disorders. Psychological Medicine, 40(9), 1495-1505. doi: 10.1017/S0033291709991942

Vieyra, M. C., Gurrola, P. G., Balcázar, N. P., Bonilla, M. M., \& Virseda, H. J. (2009). Estado de salud mental en mujeres víctimas de violencia conyugal que acuden a la Procuraduría General de Justicia del Estado de México. Psicología Iberoamericana, 17(1), 57-64. Recuperado de https://www.redalyc.org/pdf/1339/133912613007.pdf 